\title{
¿NÉMESIS OBSTÉTRICA O DISMINUCIÓN DEL RIESGO? A DEBATE BIOÉTICO EL ABORDAJE INTERVENCIONISTA EN LOS PARTOS DE BAJO RIESGO
}

\begin{abstract}
José Manuel Hernández Garre¹, Paloma Echevarría Pérez ${ }^{1}$, María José Gomariz Sandoval²
Resumen: Desde hace décadas el parto de bajo riesgo ha sido abordado, fundamentalmente en el ámbito clínico-hospitalario, desde una perspectiva intervencionista, caracterizada por la medicalización, tecnificación y mecanización asistencial. En este contexto se sitúa el presente estudio, cuyo objetivo ha sido explorar las limitaciones bioéticas de este paradigma asistencial intervencionista, mediante el análisis de su pragmatismo clínico frente a otros abordajes más humanistas. Para ello se ha llevado a cabo un profundo análisis documental en diferentes bases de datos del ámbito de las ciencias médicas y sociales, seleccionando tanto artículos de difusión científica como libros, declaraciones, estrategias y guías de práctica clínica. Los resultados muestran un patrón asistencial medicalizado, que parece incrementar las intervenciones no justificadas y con ello los efectos iatrogénicos en los partos de bajo riesgo. Se concluye que estamos ante una cultura del nacimiento que, en cierta medida, vulnera los principios bioéticos fundamentales, al subyugar la dignidad de la corporalidad femenina a intereses económicos contingentes e ideologías transhumanistas, biopolíticas y tayloristas cosificadoras.
\end{abstract}

Palabras clave: parto hospitalario, parto intervencionista, parto medicalizado, transhumanismo

\section{Obstetrics nemesis or decrease in risk? A bioethical debate on interventionist treatments in low risk childbirths}

\begin{abstract}
For decades in low-risk deliveries has been addressed, mainly in the clinical-hospital setting, from an interventionist perspective characterized by medicalization, mechanization and machining care. In this context the present study lies, whose aim was to explore the bioethical limitations of this interventionist paradigm of care through an analysis of its clinical pragmatism compared to other approaches more humanistic. For this it has conducted a thorough analysis of documents in different databases in the field of medical and social sciences, selecting both items of scientific diffusion as books, statements, strategies and clinical practice guidelines. The results show a medicalized care pattern appears to increase the iatrogenic effects in lowrisk deliveries. We conclude that this is a culture of birth which, to some, it violates basic bioethical principles to subjugate the dignity of female corporeality to contingent economic interests and transhumanists, biopolitics and taylorist ideologies reifying.
\end{abstract}

Key words: hospital birth, interventionist birth, medicalized birth, transhumanism

Nemesis obstétrica ou diminuiçáo do risco? A debate bioético a abordagem intervencionista em partos de baixo risco

Resumo: Por décadas o parto de baixo risco tem sido abordado, fundamentalmente no campo clinico-hospitalar, a partir de uma perspectiva intervencionista caracterizada pela medicalização, tecnificação e mecanização assitencial. Nesse contexto se situa o presente estudo, cujo objetivo tem sido explorar a limitaçóes bioéticas desse paradigma assistencial intervencionista através da análise de seu pragmatismo clínico frente à outras abordagens mais humanistas. Para isto, fora realizada uma profunda análise documental em diferentes bases de dados no campo das ciências médicas e sociais, selecionando tanto artigos de divulgação científica como livros, declaraçóes, estratégias e guias de prática clínica. Os resultados mostram um padrão assistencial medicalizado que parece intensificar as intervençóes não justificadas e, por conseguinte, os efeitos iatrogénicos em partos de baixo risco. Conclui-se estamos diante de uma cultura de nascimento que, em certa medida, viola os princípios bioéticos fundamentais ao subjugar a dignidade da corporalidade feminina por interesses econômicos contingentes e ideologias transumanistas, biopolíticas e tayloristas reificantes.

Palavras-chave: parto hospitalar, parto intervencionista, parto medicalizado, transumanismo

\footnotetext{
${ }^{1}$ Universidad Católica de Murcia, España

Correspondencia: jmhernandez@ucam.edu

${ }^{2}$ Hospital Universitario Virgen de la Arrixaca, España
} 


\section{Introducción}

Vivimos en un tiempo confuso, la perfección del cuerpo humano, que encumbraban los "mecanicistas" del siglo XVII, como La Mettrie, Helvetius o D'Holbach, e incluso el "biologicismo" cartesiano con el que se inauguró la Edad Contemporánea, ha dado paso hoy a la desconfianza, a la zozobra corporal. El desengaño por la corruptibilidad de nuestra propia dimensión física, el miedo a la muerte, ha engendrado la semilla del mal, y en este contexto la solución parece estar en la injerencia anatómica, en la superación de la fisiología normal. Se trata de una nueva cosmovisión en la que cobran sentido las praxis típicas de un abordaje medicalizado del nacimiento, que parece haber puesto en la intervención sus expectativas de superación de los límites naturales de la procreación. Situación que no parece marginal, sino esencia nuclear del itinerario asistencial que siguen hoy día los partos hospitalarios e intervencionistas en muchos contextos. Se trata de una nueva perspectiva de la procreación que proclama, en cierta medida, el ocaso del organismo materno tal y como lo conocemos(1), para extender el control sobre la naturaleza, con el que despuntó la ciencia moderna, a la propia naturaleza humana, "con la que se pretende hacer tanteos para averiguar sus posibilidades de mejora" (2:156). Estamos ante una nueva vuelta de tuerca en este proceso de superación de lo humano, de manera que "la noción del yo con que se inaugura la Modernidad (...) busca ahora (...) desligarse de los cauces de realización que ofrece su propia naturaleza compartida, renunciando a comprenderse desde la identidad biológica específica” (2:157). Desde estas perspectivas, parece que los clínicos, en incluso las propias madres, hubieran "identificado la naturaleza humana con la inseguridad y la vulnerabilidad por [lo que] la rechazan y no quieren identificarse con ella"(3:56). Por ello sitúan el foco de atención en el uso de la tecnología como medio de potenciación de las prestaciones de los cuerpos maternos, con la pretensión de curarlos "de una vez por todas de sus debilidades constitutivas" (4:145). Así, se pretende controlar la reproducción mediante dos mecanismos: la manipulación genética de la procreación, que supone un intento de eugenesia de lo biológico, y la manipulación tecnológicomecánica de los partos, que supone un intento de superar las propias prestaciones de la biología materna. Se trata, en definitiva, de dos abordajes que reducen los fenómenos ligados a la maternidad a simple información, de dos proyectos que ceden a las exigencias del mercado capitalista y de la tecnociencia(5). Parece que estamos, pues, ante una concepción de la corporalidad femenina en clave transhumanista que, en cierta medida, cosifica a las mujeres durante el parto ,al reducir su corporalidad a un útero-gestante que necesita de la intervención biotecnológica para traer al mundo a su bebé. Subyace, así, una concepción de la mujer que pretende superar, mediante la exaltación tecnológica, la clásica representación humanista de la corporalidad materna durante el parto en clave de empoderamiento de los propios recursos fisiológicos de la mujer.

En el marco de esta concepción de la maternidad cobra vida el patrón cultural de asistencia al nacimiento, catalogado por autores como Blázquez(6) con el sobrenombre de "parto hospitalario e intervencionista" o "parto medicalizado". Patrón que además es el más común y legitimado en los ambientes clínico-hospitalarios de los países con acceso a la tecnología sanitaria $(7,8)$. Estaríamos, en palabras de Davis-Floyd (9), ante un nuevo paradigma asistencial "tecnocrático", que ha situado el itinerario de los partos en coordenadas como la medicalización de los procesos normales, la obsesión por el control biotecnológico o la propensión a mecanizar y protocolizar todas las actuaciones clínicas(10). En el seno de este patrón asistencial del parto cobra sentido el presente estudio, cuyo objetivo ha sido explorar las limitaciones bioéticas de este modelo intervencionista del nacimiento, mediante el análisis de su pragmatismo clínico frente a otros abordajes más humanistas.

\section{Metodología}

Se realizó un análisis documental exhaustivo durante 2014, examinándose documentos pertenecientes a diversas áreas de conocimiento del ámbito de la medicina obstétrica, la antropología y la sociología. La búsqueda se realizó en bases de datos del marco de las ciencias médicas y sociales como Pudmed, CINAHl, Cochrane, Antro Base: Social and Cultural Anthropology, Current Contens, SOC-Antropología social y Periodicals Archive Online - $\mathrm{PAO}$ - En cuanto a los lí- 
mites de búsqueda, se seleccionaron únicamente aquellos artículos publicados en inglés o español en que los descriptores aparecían en el título o el abstract, no estableciéndose restricción en razón de fecha, con el objetivo de recabar la máxima información posible. Se consultaron los tesaurus de las diferentes bases de datos, y en su defecto el Medical Subject headings (MeSH), utilizándose los siguientes descriptores para las búsquedas: "posthumanism", "hospital birth", "medicalized birth" y "interventionist birth". En cuanto a los criterios de inclusión, se escogieron aquellos artículos de revistas de producción científica relacionados con la temática de la investigación, utilizándose también otros documentos de interés para el análisis documental, como libros, declaraciones, estrategias, guías de práctica clínica, programas, protocolos asistenciales e información manejada por las madres no perteneciente a los circuitos formales (información vertida por asociaciones relacionadas con la maternidad a través de páginas web y folletos no oficiales).

\section{Resultados}

Una de las primeras autoras en cuestionar el abordaje intervencionista en los partos de bajo riesgo fue la Dra. Marjorie Tew, investigadora del Nottingham University Medical School, que, tras un análisis concienzudo, concluyó que la medicalización de los partos normales, acaecida en Inglaterra en los años setenta, había supuesto un aumento de las tasas de morbimortalidad materna e infantil $(11,12)$. Tras las investigaciones de Marjorie Tew vendrían otros estudios, como los del Centro Nacional de Epidemiología en Perinatología de Oxford, el cual, tras una revisión comprensiva de la evidencia científica, comparando la seguridad del parto en contextos hospitalarios de alta intervención frente a ámbitos domiciliarios de baja intervención, determinó que no existía evidencia para apoyar la creencia de que la disminución de la mortalidad perinatal en Gran Bretaña se pudiera atribuir a la medicalización de los partos acaecida tras su hospitalización. Tampoco para confirmar que fuera más seguro para las mujeres de bajo riesgo parir en un hospital de alta intervención que en contextos de baja medicalización como los domicilios(13). En esta misma línea argumental, las investigaciones del Departamento de Medicina Social de la Universidad de Copenhague pusieron en evidencia, tras analizar 24.092 partos de varios países, que aquellos acaecidos en contextos de baja intevención presentaban menos apgars bajos, menos laceraciones severas y menos intervenciones médicas, como inducción del parto, episiotomía, fórceps y cesáreas, sin que se produjera una modificación en las cifras de mortalidad(14). Las diferentes investigaciones coincidieron en apuntar que la implantación del parto medicalizado en el seno hospitalario había aumentado las cifras de morbilidad materno-fetal, al aplicar a todas las gestantes una rutina intervencionista diseñada para mujeres de alto riesgo. Se concluyó, de esta manera, que no existía evidencias científicas que apoyaran la medicalización sistemática de todos los partos normales(15).

Tras la difusión de estos datos, en algunos países del norte de Europa, como Inglaterra, Holanda, Dinamarca o los Países Escandinavos, se produjo una drástica disminución de la medicalización en los partos de bajo riesgo. Sin embargo, en muchos contextos, como los Estados Unidos, los países europeos de la cuenca mediterránea o de Sudamérica y Asia, la medicalización injustificada de los partos normales sigue siendo aún una realidad clínica en plena vigencia(16). En este sentido, llama la atención el espectacular aumento que han experimentado las cesáreas en estos contextos, pasando de ser algo anecdótico a una realidad diaria. Las cifras son rotundas; por ejemplo en España, en 2009, los porcentajes medios ascendieron hasta un $34,6 \%$, en los centros privados, y a un $21,91 \%$ en los públicos(17), con una media total del $25,8 \%$. Lo que supone que a una de cada cuatro mujeres se le practicó una cesárea. Cifras muy elevadas si las comparamos con el $13,6 \%$ que se realizaban en los años setenta en este mismo contexto(18), momento en el que la institucionalización de los partos aún era una realidad incipiente. En otros contextos intervencionistas, como E.E.U.U., los índices son parecidos, estando alrededor del $32 \%(19)$, y en países sudamericanos, como Chile, las cifras son aún más elevadas, llegando a un $28 \%$ en los centros públicos y al $83 \%$ en los privados, con una media total de 49\% en 2010(20). A ello se suma que la tasa de cesáreas electivas en los centros privados ronda el 68\%, frente a tan solo el $12 \%$ en los públicos. Resultados muy elevados si los comparamos con los de otros países en los que 
las evidencias científicas sobre los riesgos del intervencionismo hicieron cambiar de tendencia hace unos años. Por ejemplo, en 2009, en países como Holanda, Suecia, Noruega, o Finlandia, las cifras de cesáreas se situaron entre el 15 y el 19\%(21). Muy próximas al $15 \%$ que recomienda la Organización Mundial de la Salud (OMS) como cifra máxima admisible(22).

A ello se suma, tal y como apunta la propia OMS, que al menos un tercio de estas cesáreas se llevan a cabo de forma innecesaria(23), constatándose además que, aunque en su realización influyen factores personales y sociológicos, como la situación familiar, la disponibilidad del obstetra, el miedo a las denuncias(24) o, como apunta el Nacional Institute of Health, factores no médicos (presión social, intereses económicos o rutinas de trabajo), la mayoría sigue siendo justificada bajo el paraguas de "indicaciones clínicas". Nos encontramos pues ante un nuevo fenómeno social muy consecuente, de otra parte, con la perspectiva transhumanista, lo que se ha dado en llamar el "nacimiento a la carta", y que ha convertido a esta cirugía mayor en la más común entre las mujeres(25). No se trata de un tema nimio, sobre todo si se tiene en cuenta que la cesárea es una intervención quirúrgica $y$, como tal, asociada a mayor porcentaje de complicaciones que el parto vaginal. Así, las numerosas investigaciones al respecto muestran que se asocia a una mayor mortalidad materna(26) y a un mayor índice de complicaciones, como parada cardiorrespiratoria, embolismo pulmonar, infecciones y dehiscencias en la herida $(27,28)$. Riesgos que aumentan en el caso de cesáreas repetidas, en las que se presenta el doble de mortalidad materna y un mayor riesgo de rotura uterina, placenta previa, desprendimiento placentario o embarazo ectópico(29). A ello se suman los riesgos para el bebé, que también se incrementan, aumentando la mortalidad fetal $(30,31)$ y otros problemas, como las complicaciones respiratorias y los apgars bajos(32).

Otro de los indicadores más idiosincrásicos del parto hospitalario e intervencionista es su alta incidencia de partos instrumentados que, en países medicalizados, como España, se han elevado en los últimos años hasta un 23\%(33), tasa elevada si la comparamos con la de otros países occidentales menos intervencionistas, como Inglaterra, don- de la frecuencia está entre el 10 y el 15\%(34), o los Países Nórdicos, donde las cifras están alrededor del 7\%(35). En países sudamericanos, como Chile, las tasas también son bajas, en torno a un $14 \%$, pero en estos contextos parece que las cifras no se deben tanto a una actitud expectante, sino más bien a la alta tasa de cesáreas. No se trata de un asunto insignificante, si tenemos en cuenta que el $40 \%$ de estos partos instrumentados se hacen de forma docente o profiláctica, es decir, sin que exista una indicación clara. Realizándose además el 52\% con fórceps, principal factor de riesgo de lesiones fetales, como el cefalohematoma(36), o la hemorragia intracraneal(37), y de desgarros maternos de III y IV grado que son, a su vez, la principal causa de incontinencias fecales en mujeres(33). A ello se suman otros datos epidemiológicos, como los porcentajes de episiotomías que en países medicalizados — por ejemplo, España - tienen una incidencia que oscila entre el 33,85 y el $72 \%$ en los hospitales públicos(38), alcanzando incluso en algunas clínicas privadas el 83\%. En otros países sudamericanos, como Chile, los datos son similares, encontrándose en torno al $80 \%$. Datos que si se contrastan con el $9,7 \%$ que presenta Dinamarca, el 15,8\% de Noruega o el 16,2\% de Inglaterra, resultan excesivos(21). Y que además están muy por encima de las cifras recomendadas por las evidencias científicas(39) y la propia OMS, que recomienda unas cifras entre un 10 y $20 \%(40)$.

Al margen de la disminución de los partos normales, también llama la atención una serie de rutinas médicas implantadas en los contextos intervencionistas. En esta línea cabe destacar praxis clínicas, como la administración de útero-estimulantes, el uso de la analgesia epidural o la utilización de técnicas de reproducción artificial en edades añosas de baja fertilidad. En este sentido, el uso de fármacos como la oxitocina intravenosa o las prostaglandinas vaginales, con el fin de acelerar, estimular o inducir los partos, es muy elevado en los ámbitos con un abordaje intervencionista del parto. Se estima que el uso de oxitocina exógena alcanza al 50\% de los partos de bajo riesgo, a pesar de que las recomendaciones instan a no utilizarla en caso de evolución normal(41), no estando además su administración relacionada con un acortamiento de la duración del proceso(40). En cuanto a los 
porcentajes de inducciones de parto, las estadísticas también son claras, y alcanzaron en 2009, en contextos intervencionistas como los de E.E.U.U., España o países sudamericanos, como Chile, cifras entre 22,1 y el 33,7\%(42), llegando en algunos centros privados españoles a situarse en torno al 50,3\%(43). Si contrastamos estos números con los de otros países con "culturas del nacimiento" menos intervencionistas, como Holanda, Suecia o Finlandia, las tasas disminuyen drásticamente hasta $8 \%(21)$, situándose así por debajo del $10 \%$ que recomienda la OMS(22). No se trata de una cuestión trivial, ya que las estadísticas reflejan que la inducción del parto de bajo riesgo está asociada con un aumento de los riesgos, incrementando los casos de rotura e hipertonía uterina, parto prolongado, corioamnionitis, embolia de líquido amniótico, desprendimiento de placenta, desgarros cervicales, atonía posparto, sufrimiento fetal agudo, infección neonatal, prolapso de cordón, distocia de hombros, uso de analgesia epidural y, lo que es más importante, de partos instrumentados(44) y cesáreas(45-48). Y es que, tal como afirma la OMS y las diferentes recomendaciones en materia de salud reproductiva, la inducción de parto debería realizarse únicamente en aquellos casos en los que los riesgos del parto espontáneo superasen los de una inducción: situaciones como los embarazos en vías de prolongación de más de 42 semanas de gestación, las roturas prematuras de bolsa amniótica de más de 24 horas de evolución, las enfermedades maternas graves, como la toxemia del embarazo, o las patologías del bebé que requieran atención inminente(22), evitándose asimismo en gestantes de bajo riesgo y en situaciones clínicas, tal como apunta el propio Ministerio de Sanidad y Política Social español en su "Estrategia Nacional de Salud Sexual y Reproductiva”, como la rotura de bolsa amniótica de menos de 24 horas de evolución, en crecimientos intrauterinos escasos sin insuficiencia placentaria, o incluso en casos como el embarazo en vías de prolongación sin compromiso fetal, siempre que la madre, tras ser informada de los posibles riesgos, decida esperar a que el parto se desencadene por sí solo(49).

Otra de las praxis instaurada en los contextos hospitalarios intervencionistas del parto es el uso de la epidural, técnica que ha sido más demanda desde que se adoptaron perspectivas más intervencio- nistas del parto, que incluían el uso rutinario de uterotónicos o de rotura prematura de membranas, prácticas que lógicamente convierten al parto en un proceso más doloroso(50). En este sentido, los números son irrefutables, y en contextos medicalizados, como E.E.U.U., España o países sudamericanos, como Chile, el uso de la epidural alcanza porcentajes en torno al $60 \%(51,52)$. Tasas que disminuyen en países menos intervencionistas, como Holanda con un 8\%, los Países Nórdicos entre un $16 \%$ y un $40 \%$ (53) o Inglaterra con un $20 \%(54,55)$. Pero, pese a los evidentes beneficios para las madres en términos de disminución del dolor, la administración de analgesia epidural también presenta algunos riesgos, asociándose a complicaciones como hipotensión, fiebre materna, náuseas, vómitos, prurito, retención urinaria, hemorragia posparto, dolor de cabeza por perforación de dura madre, prolongación del expulsivo, mayor uso de oxitocina y mayores tasas de parto instrumentado(56-59), realidad que se produce al interferir la epidural en algunas de las principales hormonas que se liberan en el parto, reduciendo la producción endógena de oxitocina(60-62), prostaglandinas y beta-endorfinas(63). La epidural también puede adormecer la musculatura uterina(64) y bloquear los músculos del suelo pélvico, importantes para guiar la cabeza hasta la posición idónea para nacer. Así, se ha demostrado que en las madres en las que se administra este tipo de analgesia se cuadriplica la posibilidad de presentación fetal posterior(65), lo que a su vez aumenta los riesgos de parto instrumentado(66). La epidural es, pues, tal y como señala la OMS, "uno de los ejemplos más llamativos de medicalización del parto normal, al transformar un acontecimiento fisiológico en un procedimiento médico" (40:16) que repercute, en diferentes grados, en los mecanismos biomecánicos del parto.

Otra de las realidades clínicas típicas de los contextos intervencionistas es el uso de técnicas de fertilidad como la fecundación in vitro (FIV), se trata de técnicas que permiten la gestación a madres con problemas de fertilidad, pero que también han abierto la posibilidad de ser madres a mujeres que, por su edad, se encontraban en edades infértiles. Ello, junto a otros factores socioculturales y económicos, ha hecho que hayan aumentado exponencialmente el número de gestantes añosas de 
más de 35 años, incrementándose, a su vez, los riesgos de morbilidad materna. Cuestión que no es baladí ya que las evidencias señalan una asociación entre las edades maternas avanzadas y el aumento de la incidencia de cesáreas $(67,68,69)$, partos instrumentados $(70,71,72)$, diabetes gestacional, gestosis, preeclampsia(73,74,75), placenta previa, hemorragia vaginal preparto, rotura prematura de membranas $(76,77)$ y desprendimiento de placenta(78). Riesgos que alcanzan también a los neonatos, verificándose en los recién nacidos de gestantes añosas una mayor incidencia de mortalidad neonatal inmediata, bajo peso al nacer, nacimiento prematuro $(79,80)$, cromosomapatías congénitas $(81,82)$ y test de apgar bajos(83). Nos encontramos, de esta manera, ante un contrasentido, por un lado las técnicas de fertilidad permiten superar las fronteras de la propia naturaleza femenina, posibilitado embarazos en gestantes añosas que de otra manera no se producirían, pero por otro, las propias intervenciones suponen un incremento de los riesgos maternos y fetales. Por no entrar en temas de fondo como los problemas éticos que puede suponer realizar una selección de la viabilidad fetal a través de técnicas como la selección preimplantatoria, la ecografía o las amniocentesis(84).

\section{Conclusiones}

Parece que estamos ante un abordaje de los partos que ha centrado sus expectativas en la disminución de riesgos, vertebrando para ello toda una serie de intervenciones biotecnológicas protocolizadas que pueden, paradójicamente, incrementarlos. De manera que cuando se observan de cerca los resultados clínicos del abordaje intervencionista en los nacimientos de bajo riesgo se pone en entredicho su supuesta practicidad, surgen los efectos iatrogénicos, las complicaciones que no se hubieran presentado si no se hubiesen aplicado estas praxis rutinarias, no justificadas.

Las razones que alimentan este patrón cultural del parto son múltiples, pero tienen sus matices dependiendo del contexto en el que situemos la mirada. Así, en los contextos hospitalarios privados la causa parece estar en el mercantilismo económico al que son sometidas las madres, ya que los procedimientos técnicamente complejos - como la cesárea- son mejor remunerados que los par- tos normales que se incluyen dentro de los programas obligatorios de salud, dejando a los profesionales con porcentajes de ganancia económica muy bajos. Ello explicaría que las tasas de cesáreas electivas sean tan elevadas en los centros privados frente a los públicos, cuando obviamente las indicaciones deberían ser las mismas en ambos contextos. En este habitus medicalizado el bienestar y los deseos de las madres se han ido sacrificado gradualmente en aras de intereses económicos, burocráticos y profesionales, adquiriendo la tecnología más importancia que las necesidades maternas. Sin embargo, estos argumentos no son totalmente extrapolables a los contextos públicos, donde la remuneración profesional no depende tanto del tipo de complejidad tecnológica de las intervenciones. En este ámbito la explicación habría que buscarla en la implantación de una serie de ideologías cosificadoras que representan la corporalidad materna, desde nociones transhumanistas, biopolíticas y tayloristas. Nociones desde las que el cuerpo gestante se representa como una especie de máquina sobre la que intervenir, que debe responder a unos tiempos estandarizados que den uniformidad al proceso. Se trataría, en palabras de Foucault, de una expresión de la estatalización de lo biológico, de una manifestación biopolítica por la que los Estados ejercen su biopoder de normativización de los procesos biológicos, entre ellos el parto, con el objetivo de garantizar, mediante la ciencia, la gestación de ciudadanos productivos. Estaríamos ante una especie de eugenesis estatal cuyos tentáculos lleva a normativizar las praxis sanitarias, desde la implantación de una cadena de intervenciones tecnológicas tayloristas — cesáreas, instrumentaciones, episiotomías, epiduralesbasadas en la racionalización de tiempos, hasta concepciones transhumanistas en las que la tecnología viene a solventar la supuesta fragilidad de la biología materna durante el parto. La consecuencia es el desplazamiento de la atención asistencial hacia las intervenciones y aparatos tecnológicos, hacia los datos dictados por la biometría, mientras las madres quedan cosificadas en un útero gestante concebido desde coordenadas patológicas.

Se trata de toda una cosmovisión del parto que tiene grandes peligros desde el punto de vista bioético, al no respetar el axioma, ya apuntado por Kant, por el que las madres deberían ser fines 
objetivos en sí mismas, y no medios al servicio de los intereses de terceros. Parece que estamos ante una realidad que socava la justicia, la inclusión social y el reconocimiento que merecen las madres, ante el quebranto de ciertos principios bioéticos básicos garantes de la dignidad de la madre. En este sentido, pudiéramos estar asistiendo a una especie de ruptura con el principio hipocrático por el que la primera norma de cualquier intervención clínica es no generar efectos nocivos. Se trataría, en palabras de Illich, de una especie de "contraproductividad" por la que los organismos de las sociedades industriales, entre ellos los de la asistencia sanitaria hospitalaria, se van alejando del fin para el que fueron creados conforme aumentan en tamaño y complejidad, convirtiéndose en engranajes viciados que no solo responden a los intereses de las madres, sino a toda una serie intereses económicos, profesionales y biopolíticos(84). En este sentido, las estructuras clínicas y los itinerarios asistenciales del parto normal no parecen ser una excepción, y cuando se mercantilizan y medicalizan pueden convertirse involuntariamente en sistemas generadores de efectos iatrogénicos. En esta misma línea, la excesiva intervención y medicalización de los partos normales rompe con el principio de autonomía de las madres, con el derecho de darse a sí mismas las normas y reglas a seguir durante los mismos, al cosificarlas en una especie de útero-gestante que se convierte en diana de toda una cadena de intervenciones tayloristas.
Parece, pues, que sería necesario articular nuevas praxis clínicas menos medicalizadas en el parto de bajo riesgo, de manera que en los contextos intervencionistas se pudiera armonizar las necesidades institucionales y profesionales con la seguridad materno-fetal. En este sentido, habría que desarrollar nuevos itinerarios clínicos para los partos de bajo riesgo, que respetasen en mayor medida los procesos y evoluciones normales, a la vez que se mantienen los estándares de seguridad. Ello supondría una inevitable revisión de las nociones ideológicas y mercantilistas que en la actualidad vertebran los procesos ligados a la maternidad en muchos contextos.

\section{Agradecimientos}

A la Universidad Católica de Murcia y al Grupo de Investigación "Pensamiento en el contexto social" de dicha Universidad el apoyo prestado durante la realización del artículo. 
¿Némesis obstétrica o disminución del riesgo? - José Manuel Hernández, Paloma Echevarría, María José Gomariz

\section{Referencias}

1. Caronia A. Il cyborg. Saggio sull'uomo artificial, Shake; 2001.

2. Ferrer U. Posthumanismo y dignidad de la especie humana. En: Ballesteros J, Fernández E, eds. Biotecnología y Posthumanismo. Navarra: Aranzadi; 2007: 153-170.

3. Viola F. La defensa e la persona humana en la era tecnológica. En: Ballesteros J, Fernández E, eds. Biotecnología y Posthumanismo. Navarra: Aranzadi; 2007; 47-66.

4. Saraceni G. El cuerpo del delito. Reflexiones jurídico-filosóficas sobre el posthumanismo. En: Ballesteros J, Fenández E, eds. Biotecnología y Posthumanismo. Navarra: Aranzadi; 2007; 139-152.

5. Ballesteros J. Biotecnología, Biolítica y Posthumanismo. En: Ballesteros J, Fernández E, eds. Biotecnología y Posthumanismo. Navarra: Aranzadi; 2007; 21-46.

6. Blázquez M. Ideologias y prácticas de género en la atención sanitaria del embarazo, parto y puerperio: el caso del área 12 de la Comunidad de Madrid. Tesis Doctoral. Tarragona: Universidad Rovira i Virgili; 2009.

7. Montes MaJ. Medicalización reproductiva o la alienación del cuerpo. Enfermería clínica 1999; 9(5): 206-212.

8. Martin E. The Woman in the Body. Boston: Beacon Press; 1987.

9. Davis-Floyd R. The technocratic, humanistic, and holistic paradigms of childbirth. International Journalof Gynecology \& Obstetrics 2001; 75(1): 5-23.

10. Hernández JM, Echevarría P. La parte negada del parto institucionalizado. Explorando sus bases antropológicas. Revista de Dialectología y Tradiciones Populares 2014: 69(2): 327-348.

11. Tew M. Place of birth and perinatal mortality. The Journal of the Royal College of General Practitioners 1985: 35: 390-394.

12. Tew M. Safer childbirth: a Critical History of Maternity Care. New York: Oxford University Press; 1990.

13. Campbell R, Macfarlane A. Where to be born? Oxford: National Perinatology Unit; 1987.

14. Olsen O. Meta-analysis of the safety of Home Birth. Birth 1997; 24(1): 4-13.

15. Thomson A. ¿Existe la evidencia científica para la medicalización de los cuidados maternales? MIDIRS, Midwifery Digest 2000; 10(4): 416-420.

16. Wagner M. Born in the USA: How a broken maternity system must be fixed to put women and children first. Berkeley: University of California Press; 2006.

17. Ministerio de Sanidad y Política Social. Indicadores Clave del Sistema Nacional de Salud. Madrid: . INCLASNS-BD MSPS; 2010.

18. Martínez-Frías M, Bermejo E, Rodríguez-Pinilla E, et al. Evolución secular y por autonomías de la frecuencia de tratamientos de fertilidad, partos múltiples y cesáreas en España. Medicina Clínica 2005; 124: 132-139.

19. Declerq E, Sakala C, Corry MP, et al. Listening to mothers II: report of the first national survey of women's childbearing experiences. The Journal Perinatal Education 2007; 16(4): 9-14.

20. Murray SF. Relation between private health insurance and high rates of caesarean section in Chile: qualitative and quantitative study. British Medical Journal 2000; 321: 1501-1505.

21. Europeristat. European Perinatal Health Report. Europeristat Project; 2009.

22. Organización Mundial de la Salud. Declaración de Fortaleza. Tecnología apropiada para el parto. Ginebra: OMS; 1985.

23. Arrieta A, García Prado A. El incremento de las cesáreas en los hospitales públicos: Un análisis de la capacidad hospitalaria y de los incentivos médicos. Economía de la Salud 2012; 73: 13-16.

24. Cobo D. Estrategias para disminuir la tasa de cesáreas en nuestro medio. Servicio de obstetricia y Ginecológica Hospital Virgen de las Nieves. Granada; 2011.

25. Mathai M. y Hofmeyr G.J. Incisiones quirúrgicas abdominales para la cesárea (Revisión Cochrane traducida). La Biblioteca Cochrane Plus; 2008, 4.

26. Shearer EL. Cesarean section: medical benefits and cost. Social Sciencia Medicine 1993; 37(10): 1223-1231.

27. Anaya-Prado R, Madrigal-Flores R, Reveles-Vázquez S, et al. Morbilidad materna asociada a operación cesárea. Cirugía $y$ cirujanos 2008; 76(6): 467-472.

28. Cárdenas R. Complicaciones asociadas a la cesárea: la importancia de un uso módicamente justificado. Gaceta Médica de México 2002; 138(4): 357-366.

29. Hemmsinki E, Marilainen J. Long-term effects of cesarean sections: ectopic pregnancies and plancent problems. American Journal of Obstetrics and Gynecology 1996; 174(5): 1569-1574.

30. MacDorman MF, Menacker F, Declercq E. Cesarean birth in the United States: epidemiology, trends and outcomes. Clinic Perinatology 2008; 35(2): 293-307. 
31. MacDorman MF, Declercq E, Menacker F, et al. Infant and neona-tal mortality for primary cesarean and vaginal births to women with "no indicated risk", United States, 1998-2001 birth cohorts. Birth 2006; 33(3): 175-182.

32. Benterud T, Sandvik L, Lindemann R. Cesarean section is associated with more frequent pneumothorax and respiratory problems in the neonate. Acta Obstetrica et Gynecologica Scandinavica 2009; 88(3): 359-361.

33. Aceituno-Velasco L. Tasa de partos instrumentales en España. Progresos de Obstetricia y Ginecología 2009; 52(10): 609615.

34. Royal College of Obstetricians and Gynaecologists of Ingland. Operative vaginal delivery. Guideline 2005; 26.

35. Stakes. Official Statistics of Finland. Parturients, births and newborns - preliminary data for 2006; 2006.

36. Johnson JH, Figueroa R, Garry D, et al. Immediate Maternal and Neonatal Effects of Forceps and Vacuum-Assisted Deliveries. Obstetric \& Gynecology 2004; 103: 513-518.

37. Jhawar BS, Ranger A, Steven D, et al. Risk Factors for Intracranial Hemorrhage Among Full-Term Infants: A CaseControl Study. Neurosurgery 2003; 52: 581-590.

38. Ministerio de Sanidad y Consumo. Estrategia para la atención al parto normal. Madrid: MSC; 2007.

39. Carroli G, Belizán J. Episiotomía en el parto vaginal. (Revisión Cochrane traducida). La Biblioteca Cochrane plus, 2008; 4.

40. Organización Mundial de la Salud. Cuidados en el parto normal: una guía práctica. Ginebra: OMS; 1996.

41. Ministerio de Sanidad y Política Social. Guía de Práctica Clínica sobre la atención al parto normal. Madrid: MSPS; 2010.

42. Caughey AB, Sudaram V, Kaimal AJ, et al. Maternal and neonatal outcomes of elective induction of labor. Evidence Report/Technology Assessment 2009; (176): 1-257.

43. Generalitat Valenciana. Conselleria de Sanitat. Salud Reproductiva. Valencia: Generalitat Valenciana; 2009.

44. Dublin S, Lydon-Rochelle M, Kaplan RC, et al. Maternal and neonatal outcomes after induction of labor without an identified indication. American Journal of Obstetrics and Gynecology 2000; 183(4): 986-994.

45. Maslow AS, Sweeny AL. Elective induction of labor as a risk factor for cesa-rean delivery among low-risk women at term. Obstetrics \& Gynecology 2000; $95(6$ Pt 1): 917-922.

46. Moore LE, Rayburn WF. Elective induction of labor. Obstetricts \& Gynecology 2006; 9(3): 698-704.

47. Michelson KA, Carr DB, Easterling TR. The impact of duration of labor induc-tion on cesarean rate. Obstetricts \& Gynecology 2008; 199(3): 299-304.

48. Ennen CS, Bofill JA, Magna EF, et al. Risk factors for cesarean delivery in preterm, term and posterm pa-tients undergoing induction of labor with an unfavorable cervix. Gynecologic and Obstetric Investigation 2009; 67(2): 113-117.

49. Ministerio de Sanidad y Política Social. Estrategia Nacional de Salud Sexual y Reproductiva. Madrid; MSPS; 2010.

50. Towler J, Bramall J. Midwives in History and Society. Beckenham: Croom Helm; 1986.

51. Sakala C, Declerq E, Corry M. Listening to mothers: report of the first national survey of women's childbearing experiences. Journal of Obstetric, Gynecologic, \& Neonatal Nursing 2002; 31(6): 633-634.

52. Junta de Andalucía. Consejería de Salud y Bienestar Social. Partos con analgesia epidural. Andalucía por grupos de edad, 200-2010. Porcentaje. Andalucía: Junta de Andalucía; 2012.

53. Ekéus C, Cnattingius S, Hiern A. Epidural analgesia during labor among inmigrant women in Sweden. Acta Obstetricia et Gynecologia Scandinavica 2010, 89(2): 243-249.

54. Khor LJ, Jeskins G, Cooper GM, et al. National obstetric anaesthetic practice in the UK 1997/1998. Anaesthesia 2000; 55(12): 1168-1172.

55. Department of Health. Statistical Bulletin-NHS Maternity Statistics, England: 2003-2004. London, UK: Department of Health Statistical; 2004.

56. Mayberry LJ, Clemmens D, Anindya D. Epidural analgesia side effects, co-interventions, and care of women during childbirth: a systematic review. American Journal Obstetrics \& Gynecology 2002; 186(5 Suppl Nature): S81-93.

57. Leighton BL, Halpern SH. The Effects of Epidural Analgesia on Labor, Maternal, and Neonatal Outcomes: A Systematic Review American Journal Obstetrics \& Gynecology 2002; 186 (5 Supplement Nature): S69-77.

58. Goetzl LM. Obstetric analgesia and anesthesia. Obstetric and Gynecology 2002: 100: 177-191.

59. Anim-Somuah M, Smyth R, Howell C. Epidural versus non-epidural or no analgesia in labour. (Cochrane Review). La Biblioteca Cochrane Plus, No 4, Oxford; 2008.

60. Goodfellow CF, Hull MGR, Swaab DF, et al. Oxytocin Deficiency at Delivery with Epidural Analgesia. British Journal of Obstetrics Gynaecology 1983; 90: 214-219.

61. Stocche RM, Klamt JG, Antunes-Rodrigues J, et al. Effects of Intrathecal Sufentanil on Plasma Oxytocin and Cortisol Concentrations in Women During the First Stage of Labor. Regional Anesthesia and Pain Medicine 2001; 26: 545-550. 
62. Rahm VA, Hallgren A, Högberg H, et al. Plasma Oxytocin Levels in Women During Labor With or Without Epidural Analgesia: A Prospective Study. Acta Obstetricia et Gynecol ogica Scandinavica 2002; 81: 1033-1039.

63. Behrens O, Goeschen K, Luck HJ, et al. Effects of Lumbar Epidural Analgesia on Prostaglandin F2 Alpha Release and Oxytocin Secretion During Labor. Prostaglandins 1993; 45: 285-296.

64. Arici G, Karsli B, Kayacan, N, et al. The Effects of Bupivacaine, Ropiva-caine and Mepivacaine on the Contractility of Rat Myometrium. International Journal of Obstetric Anesthesia 2004; 13: 95-98.

65. Lieberman E, Davidson K, Lee-Parritz, A, et al. Changes in Fetal Position During Labor and their Association with Epidural Analgesia. Obstetric and Gynecology 2005; 105: 974-982.

66. Ponkey SE, Cohen AP, Heffner LJ, et al. Persistent Fetal Occiput Posterior Position: Obstetric Outcomes. Obstetric and Gynecology 2003; 101: 915-920.

67. Grimes D, Gross GK. Pregnancy otucomes in blak women aged 35 and older. Obstetric and Gynecology 1981; 58(5): 614-620.

68. Ziadeh SM. Maternal and perinatal outcome in nulliparous women aged 35 and older. Gynecologic and Obstetric Investigation 2002; 54(1): 6-10.

69. Studzinski, Z. Pregnancy and delivery in women over 40 years old. Wiad Lek 2004; 57(3-4): 140-144.

70. Seufert R, Casper F, Krass A, et al. The older primiparan obstetrical risk group. Zentralbl Gynakol 1994; 116 (3): $169-$ 172.

71. Gilbert WM, Nesbitt TS, Danielsen B. Childbearing beyond age 40: pregnancy outcome in 24.032 cases. Obstetric and Gynecology 1999; 93(1): 9-14.

72. Roman H, Robillard PY, Julien C, et al. Pregnancy beyond age 40 in 382 women: a retrospective study in Reunion Island. Journal de Gynecologie Obstetrique et Biologie de la Reproduction 2004; 33(7): 615-622.

73. Heimann F, Messerer D, Baltzer J. Pregnancy, labor and fetal outcome in fe-males over 40 years of age. Geburtshilfe Frauenheilkd 1993; 53(6): 411-415.

74. Miletic T, Aberle N, Mikulandra F, et al. Perinatal outcome of pregnancies in women aged 40 and over. Collegium Antropologicum 2002; 26(1): 251-258.

75. Jacobsson B, Ladfors L, Milsom I. Advanced maternal age and adverse perinatal outcome. Obstetric and Gynecology 2004; 104(4): 727-733.

76. Mikulandra F, Perisa M, Merlak I, et al. Pregnancy and delivery in women aged 35 year and over. Zentralblatt fur. Gynakologie 1993; 115(4): 171-176.

77. Bianco A, Stone J, Lynch L, et al. Pregnancy outcome at age 40 and older. Obstetric and Gynecology 1994; 87(6): $917-$ 922.

78. Holger EO, Smythe AR. Pregnancy in women over forty. Obstetric and Gynecology 1977; 49(3): 257-261.

79. Viegas OA, Leong W.P, Ahmed S, et al. Obstetrical outcome with in-creasing maternal age. Journal of Biosocial Science 1994; 26(2): 261-267.

80. Salihu HM, Shumpert MN, Slay M, et al. Chilbearing beyond maternal age 50 and fetal outcomes in the United States. Obstetric and Gynecology 2003; 102(5Pt1): 1006-1014.

81. Caspi E, Lifshitz Y. Delivery at 40 years of age and over. Journal of Medical Sciences 1979; 15(5): 418-421.

82. Milner M, Barry-Kinsella C, Unwin A, et al. The impact of maternal age on pregnancy and its outcome. International Journal of Gynaecology \& Obstetrics 1992; 38(4): 281-286.

83. Ezra Y, Mcparland P, Farine D. High delivery intervention rates in nullipa-rous women over age 35. European Journal of Obstetrics \& Gynecology and Reproductive Biology 1995; 62 (2): 203-207.

84. Rapp R. Testing women, testing the fetus. The social impact of Amniocentesis in America. London: Routledge; 1999.

85. Illich I. Némesis Médica. México DF: Joaquín Mortiz; 1978.

Recibido: 12 de octubre de 2015

Aceptado: 23 de enero de 2016 